\title{
REGENERATION FOLLOWING DENERVATION OF MINCED GASTROCNEMIUS MUSCLES IN MICE
}

\author{
SHAHZAD A. MUFTI* \\ Department of Anatomy, University of Michigan, Ann Arbor, Mich. 48104 (U.S.A.) \\ (Received 3 February, 1977)
}

\section{SUMMARY}

Gastrocnemius muscles in mice were minced and orthotopically implanted. At the same time all nervous supply to this muscle was completely removed. It was observed that initially the pattern of muscle regeneration was similar to what was observed in a normally innervated implant. But by day 6 , distinct degeneration of regenerated muscle fibres sets in, which continues unabated so that by about 3 weeks there usually remains only a thin band of connective tissue in place of the implant.

Histochemically, there is a gradual loss of SDH, myofibrillar ATPase and cholinesterase activities within the degenerating muscle fibres and a corresponding appearance of these enzymes in the regenerating fibres. In the denervated implants, with the onset of degeneration of the regenerating fibres, the enzymatic activities were also lost. Histochemical fibre typing was not achieved within the regenerating fibres.

The regeneration and degeneration pattern of the denervated muscle observed in the present study is compared with the one observed in other animals.

\section{INTRODUCTION}

It has now been established that nerves intimately control the structure and function of muscle. Through many experimental studies, it has been shown that nerves influence the structural integrity of the muscle (Gutmann and Zelena 1962), its histochemical characteristics (Guth, Samaha and Albers 1970; Burke, Levine, Fajac, Tsairis and Engel 1971) and its physiological properties such as speed of contraction (Buller, Eccles and Eccles 1960; Close 1964). The best example of a nerve influence is

This work was done during the tenure of a Postdoctoral fellowship by the Muscular Dystrophy Associations of America, Inc.

* Present address: Department of Zoology, University of the Punjab, New Campus, Lahore, Pakistan. 
provided by cross-innervation experiments (Buller et al. 1960; Guth et al. 1970: Samaha, Guth and Albers 1970) in which enzyme profile and speed of muscle are reversed or modified according to the type of innervation.

Although nerves play an important role in the ontogenetic development of muscle, especially in terms of its growth and maturation, there is a known intrinsic developmental capacity of muscle which does not depend on nerves (Harrison 1904; Hamburger 1939; Hughes 1968). Recently the regenerating muscle system has been extensively used for the study of the nerve-muscle relationship. In this system, the muscle, after being thoroughly traumatized by mincing, undergoes a regenerative process which in many respects is similar to its embryonic development (Carlson 1973).

The influence of the nerves during early phases of muscle regeneration has recently been studied in several species such as rat (Mong 1975), frog (Hsu 1974) and mouse (Yeasting 1969). In these studies there is considerable variation reported in terms of both the length of time a muscle can continue regenerating without a nerve supply and the degree of its differentiation in the absence of the nerves. For example, according to Studitsky (1963), muscle regeneration does not occur to any appreciable extent without a nerve supply. Zhenevskaya (1962) reported early muscle regeneration up to myotube formation whereas Hsu (1974) reported formation of almost mature muscle fibres in the absence of nerves, followed by their degeneration and finally elimination by day 30 . Mong (1975) made similar observations in rats, although time for the onset of muscle breakdown varied. Yeasting (1969), while working on mice, described no histological differences in the regenerative processes in normally innervated and denervated gastrocnemius muscle during the 5 -week period of investigation. In most of these studies only gross morphological and histological observations were. made and there was no correlation made in terms of histochemical differentiation of muscle.

The present investigation was initiated to study the effects of denervation on muscle regeneration, especially of the degree of muscle development achieved and the time course for the breakdown of regenerated muscle as compared to other species studied. Histochemical maturation of the regenerating fibres was also considered as well as the time of innervation and formation of motor end-plates.

\section{MATERIAL AND METHODS}

Commercially obtained male Swiss Webster mice (20-35 g) were used in this series of experiments. They were anaesthetized with ether. The mincing and orthotransplantation of the gastrocnemius muscle was performed in the manner described by Carlson (1968) for rats. In the experimental series, the three branches of the sciatic nerve supplying the gastrocnemius muscle, namely peroneal, sural and anterior tibial, were transected proximally and removed all the way to the distal end. The transected end of the sciatic nerve, which lay underneath the biceps femoris muscle, was turned proximal and sutured to the underlying tissue so as to eliminate nerve fibre growth distally towards the implant. In the control series, these nerves were retained intact, although their connections with the muscle are obviously broken at the time of removal of the muscle. 
After the operation the animals were kept singly in a cage and fed ad libitum. At certain prescribed regular intervals the animals were anaesthetized again. The implants were removed, stretched on to a piece of filter paper and fixed in Bouin's fixative. These were then processed, sectioned and stained with haematoxylin and eosin.

For histochemical analysis, the belly portion of the implant was frozen in isopentane which was cooled to $-40^{\circ} \mathrm{C}$ with dry ice. These were then sectioned at $-20^{\circ} \mathrm{C}$ in a Cryostat. The sections were air-dried for $1 / 2-1 \mathrm{hr}$ and stained for succinic dehydrogenase (SDH), myofibrillar ATPase and cholinesterase by the methods described by Nachlas, Tsou, deSouza, Cheng and Seligman (1957), Padykula and Herman (1955) and Henderson's modification of Gomori's method (Pearse 1972) respectively.

\section{RESULTS}

Histology

\section{Control series}

The degeneration of the minced implanted muscle fibres starts from the periphery and proceeds towards the center. After 2 days there is a distinct rim of degenerating fibres towards the periphery. The morphology of the degenerating fibres is typical. The sarcoplasm becomes deeply eosinophilic and vacuolated while the nuclei become pyknotic and undergo fragmentation. By the 4th day, within this mass of degenerating fibres, a few myogenic cells arise, which are seen aligning themselves in longitudinal rows or at some other places they even fuse to form myotubes (Fig. 1). By the 6th day, about $50 \%$ of the total area, towards the periphery, is occupied by

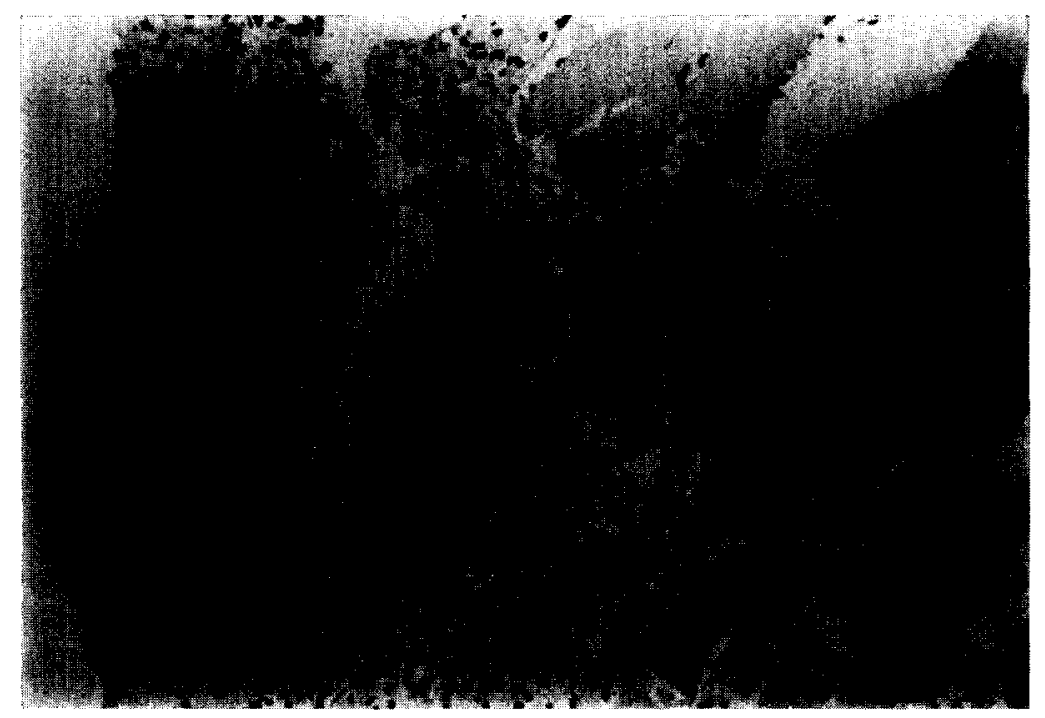

Fig. 1. A 4-day control implant. Note early myotube formation towards the periphery (arrow). $\mathrm{H}-\mathrm{E}, \times 100$. 


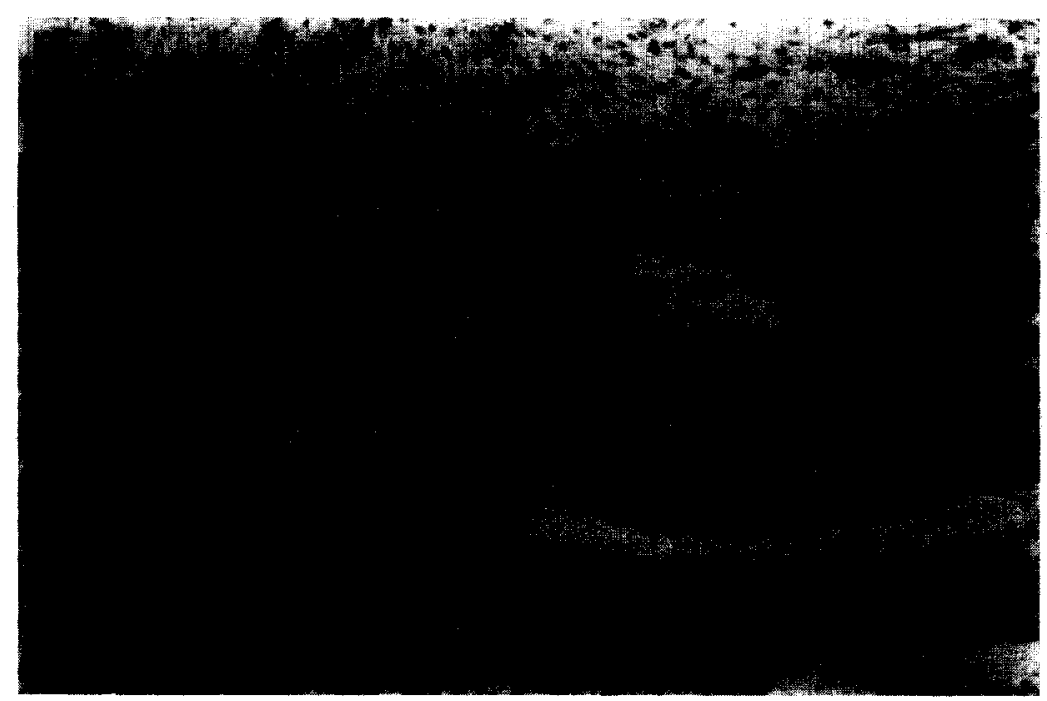

Fig. 2. Peripheral regenerating muscle fibres in a 6 -day control implant. $H-E, \times 100$.

many regenerating myotubes (Fig. 2). These myotubes have become well differentiated and many of them develop striations. The nuclei are still centrally located in these myotubes. The tendinous connections of the regenerating muscle, especially towards the distal end, become well developed. The vascularization is also conspicuous in relation to the regenerating myotubes. The ischaemic, degenerating original muscle fibres persist in the central regions of the implant until about 15-18 days. By this time, most of the regenerated muscle fibres have differentiated into mature-looking muscle fibres with distinct striations, but characteristically, in most of these, the nuclei still lie

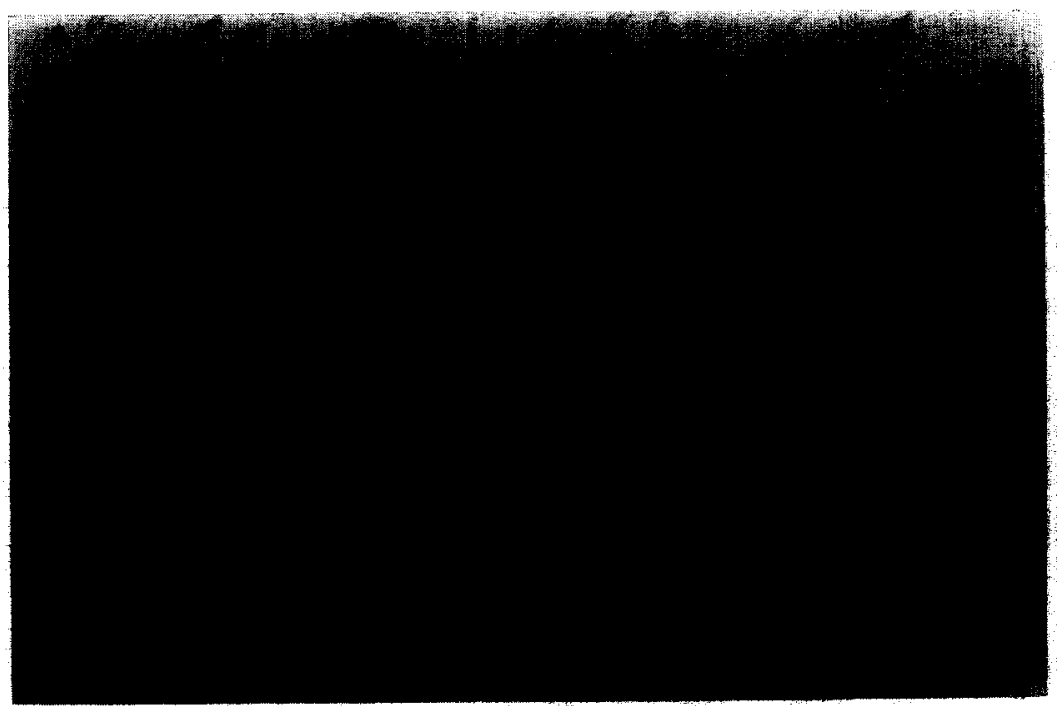

Fig. 3. Higher magnification of a few of the muscle fibres in a 15-day control regenerate. Note that myonuclei still lie centrally in most of the cases (arrow). H-E, $\times 250$. 


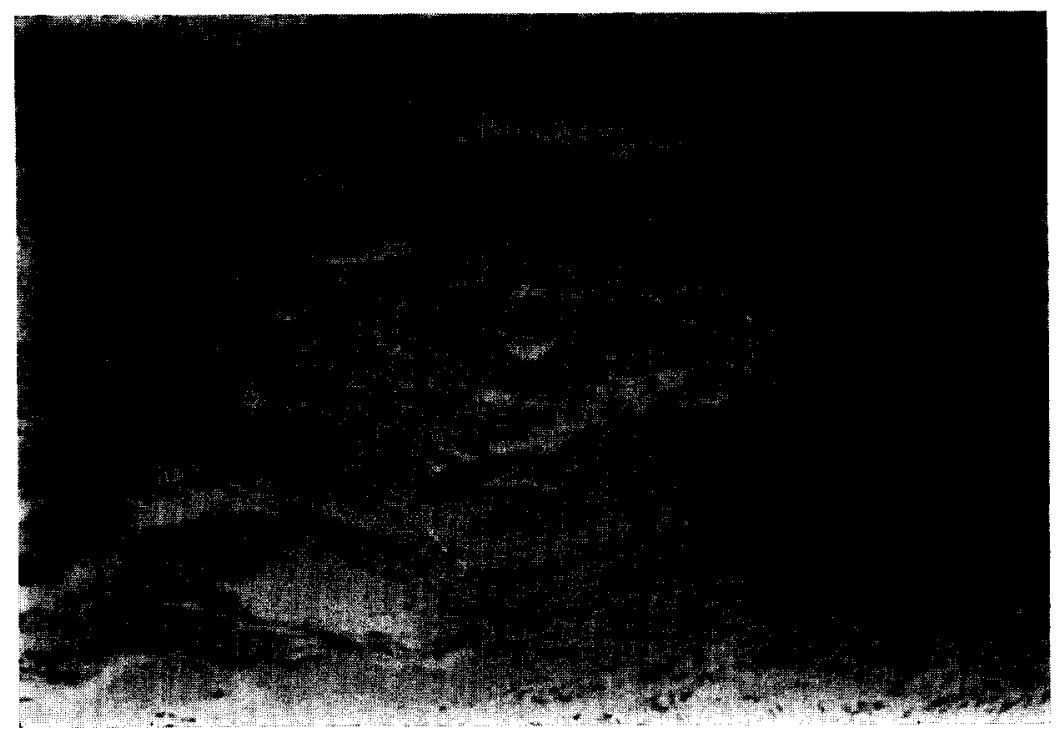

Fig. 4. Mature-looking regenerated muscle fibres in a 30-day control implant. H-E, $\times 100$.

centrally (Fig. 3). Their orientation has also become quite regular and they lie parallel to the longitudinal axis. The tendons have also grown tremendously, and most of the distal part of the regenerate is nothing but the huge calcaneal tendon. By 30 days, the muscle fibres grow further and look quite mature in their morphological characteristics. Many of the fibres have peripheral nuclei. From this stage onwards (Fig. 4), until day 70, there is further growth and development on this basic pattern. The muscle fibres continue to develop both structurally and functionally but the overall size of the regenerate remains small and strap-like and does not reach even half the mass of the original muscle.

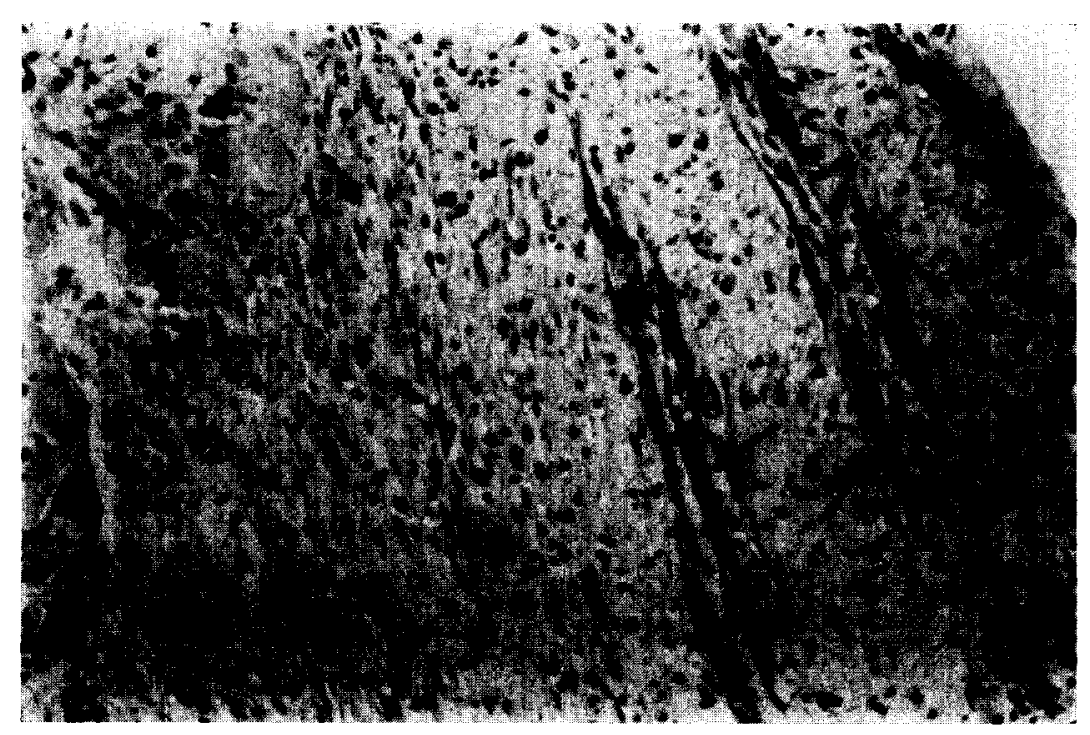

Fig. 5. A portion of a 4-day denervated implant. The myotube formation has initiated towards the peripheral region (arrow). H-E, $\times 100$. 


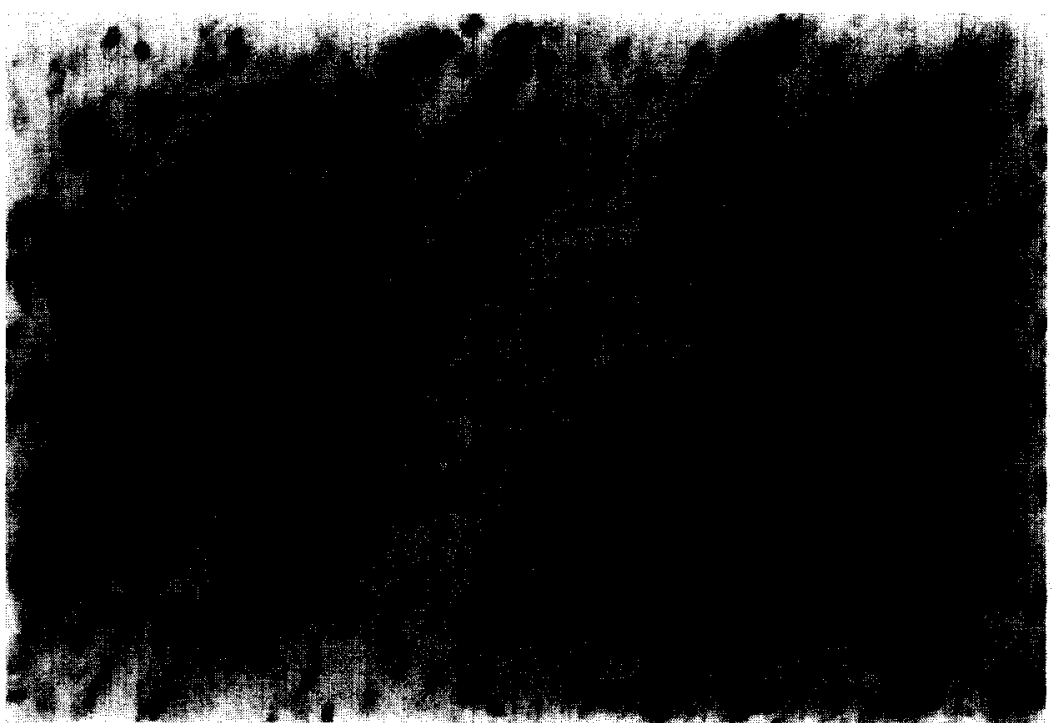

Fig. 6. A higher magnification of the degenerating myotubes seen in a 6-day denervated implant. Note the clumping of the myonuclei. H-E, 250.

\section{Denervated series}

During the first 24 days, the general picture is similar to that of the controls. There is the beginning of myoblast differentiation and myotube formation by the 4th day (Fig. 5); but very soon, that is by 6 days, quite distinct degeneration of the regenerated myotubes sets in (Fig. 6). By this day, many myotubes can be seen to be affected. Their sarcoplasm undergoes partial dissolution or becomes shriveled up in many cases. The nuclei become pyknotic and clump together. By 7-10 days almost all of the

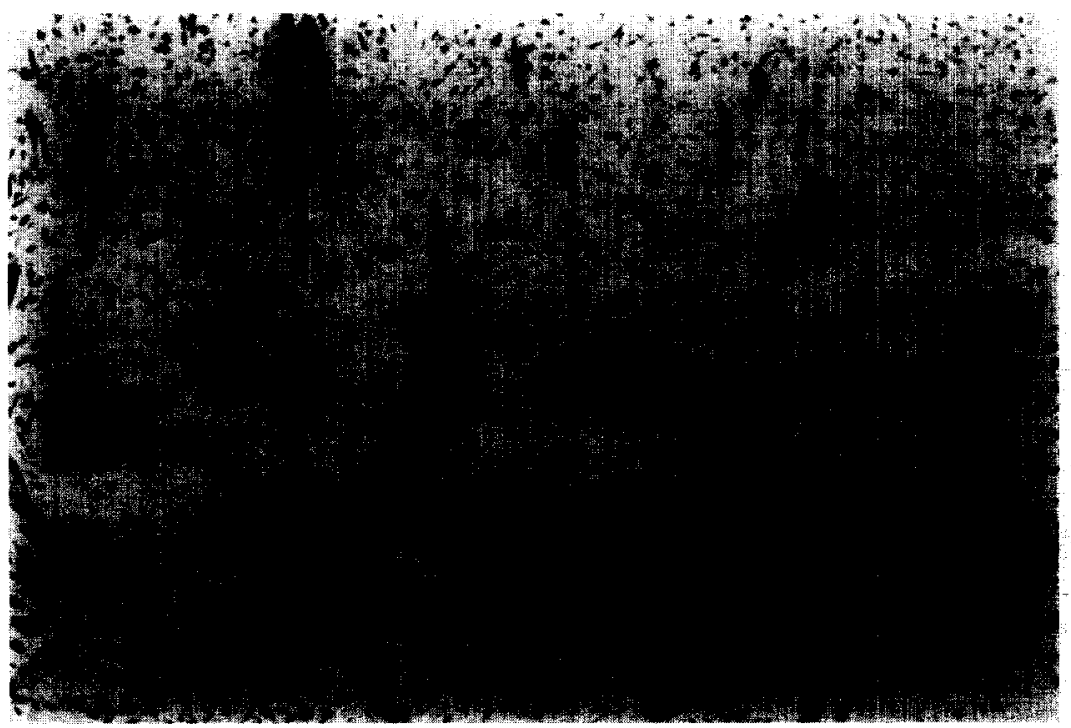

Fig. 7. Degenerating myotubes in a 7-day denervated implant. H-E, $\times 100$. 


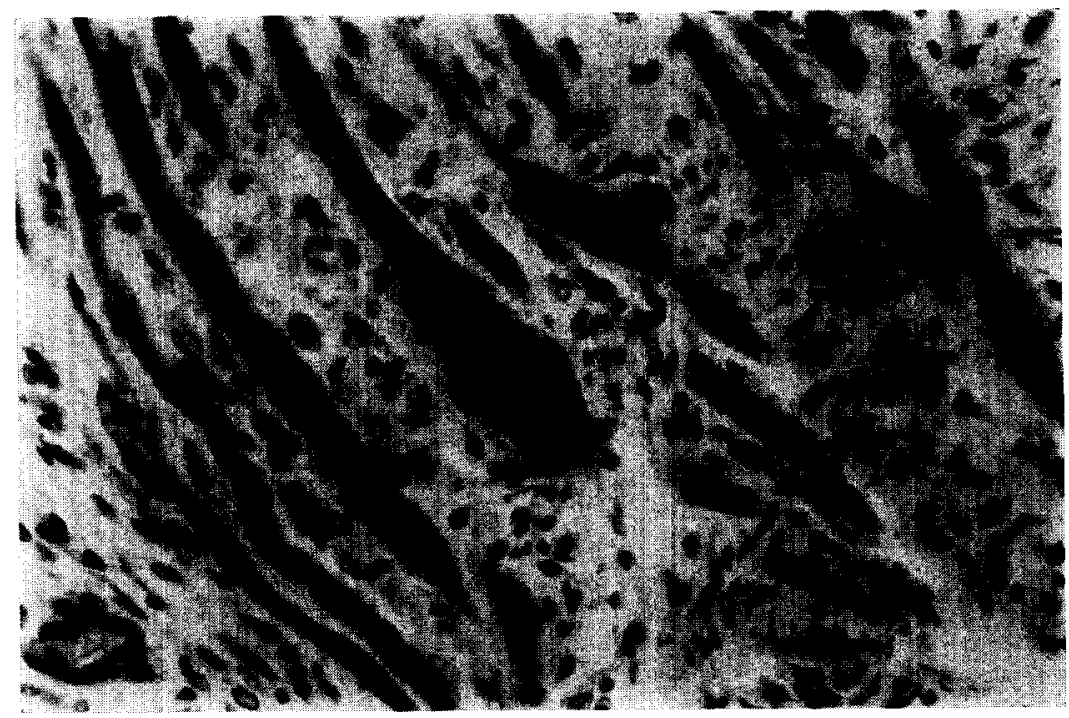

Fig. 8. A few of the degenerating muscle fibres in a 10-day denervated implant. Note the packing together of disc-shaped, highly pyknotic nuclei, a condition typical of denervation-induced atrophy and ultimate degeneration of muscle fibres. H-E, $\times 250$.

regenerated myotubes show typical degenerative changes (Figs. 7 and 8), in the form of sarcoplasm dissolution and nuclear clumping and disintegration. Connective tissue and adipose tissue are much more than in the control series. This disintegration and consequent elimination of the regenerating fibres continues until about days 15-18 (Fig. 9). By day 20 there is usually hardly any trace of the regenerating muscle left.

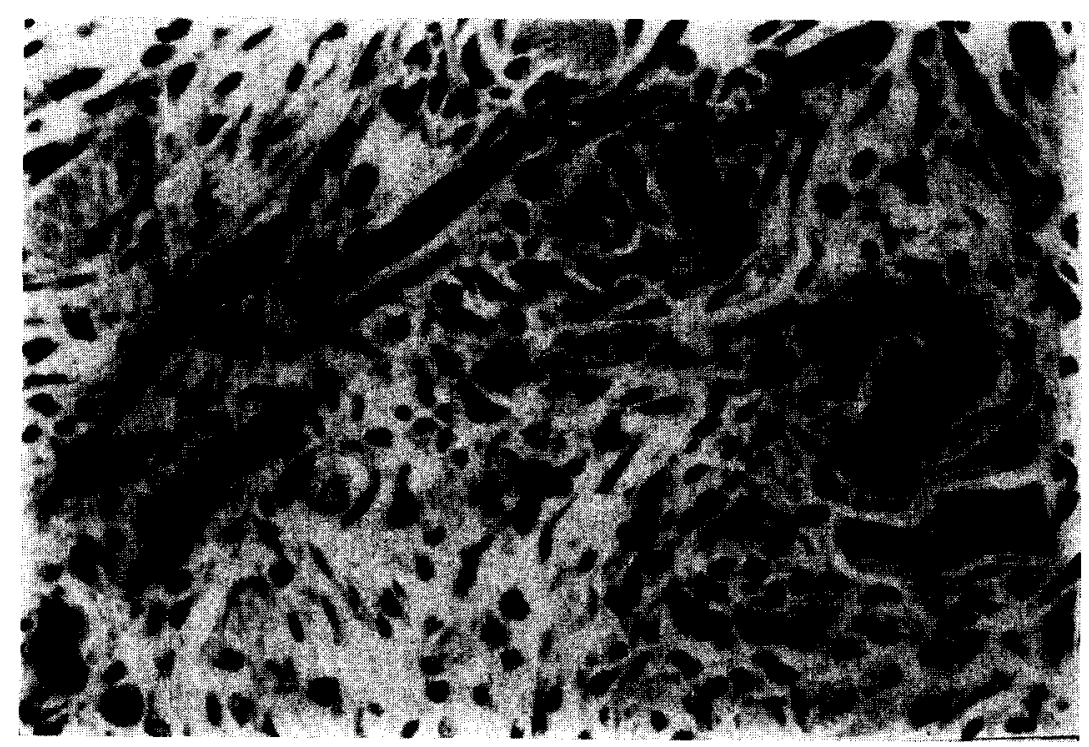

Fig. 9. A higher magnification of the degenerating muscle fibres in a 15-day denervated implant. Note that the fibres are at the brink of complete elimination. H-E, $\times 250$. 


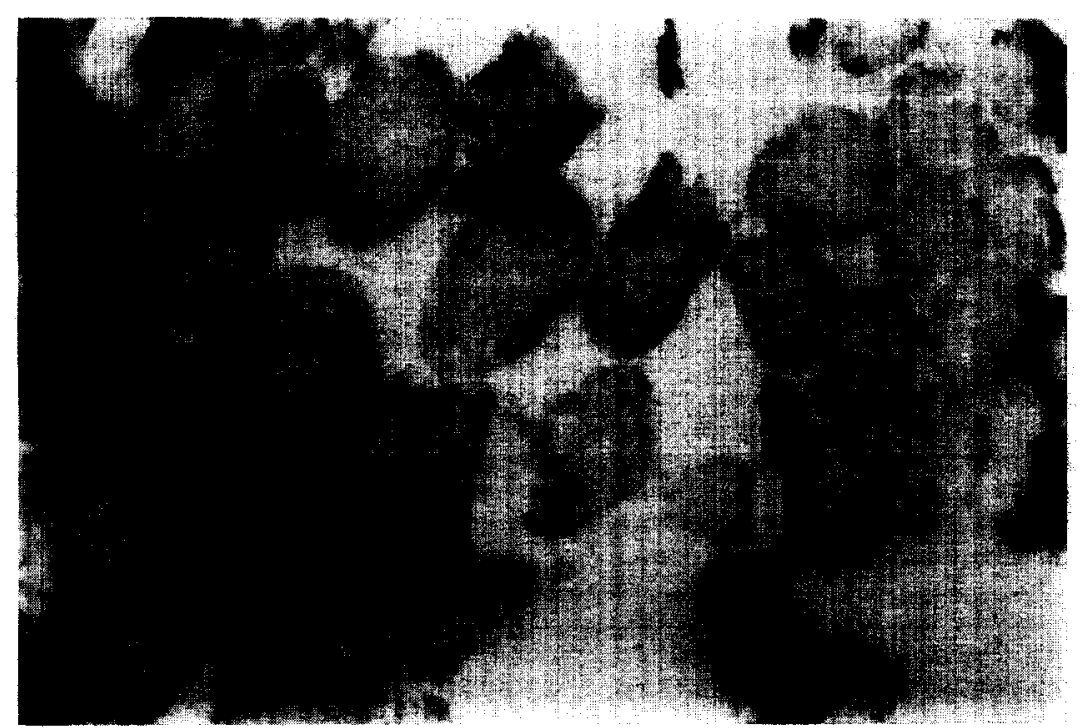

Fig. 10. SDH activity in a few fibres (arrow) in a 4-day control implant. 100.

The whole mass may be resorbed to a great extent, leaving only a band of connective and adipose tissue.

\section{Histochemical observations}

\section{Control series}

$S D H$. Histochemical evidence of SDH activity persists in few of the fibres to the 4th day although the majority of them have already lost it (Fig. 10). By 8-10 days

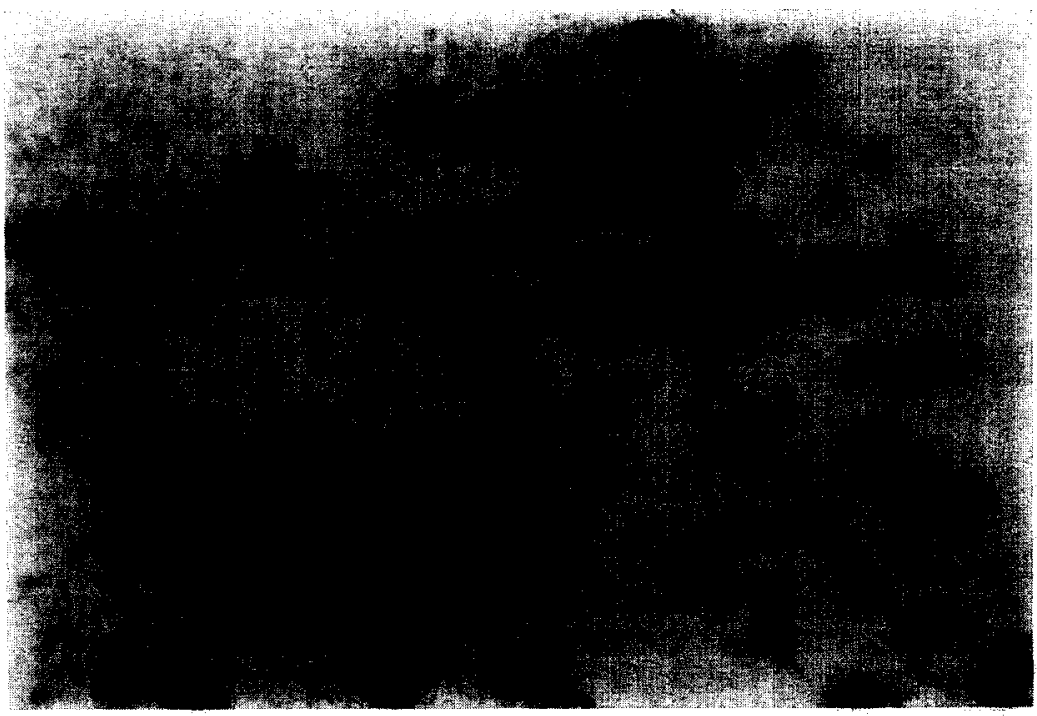

Fig. 11. SDH-positive myotubes in an 8-day control. $\times 100$. 


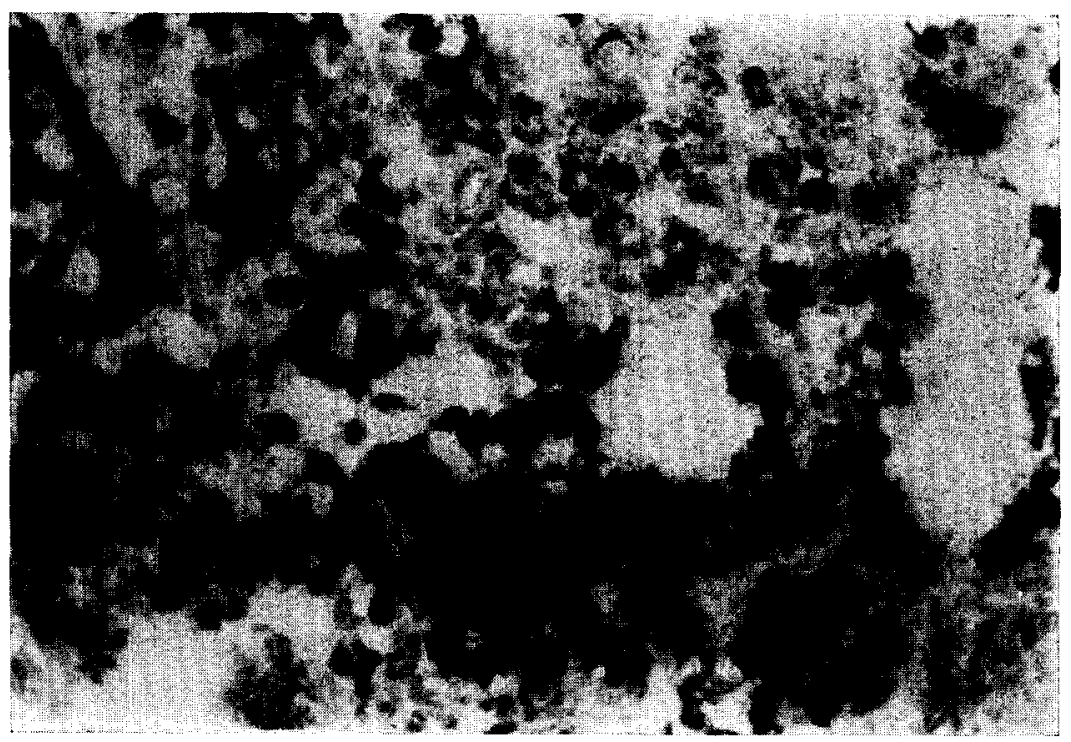

Fig. 12. SDH activity pattern in a 30 -day control implant. Note that fibre typing has become quite conspicuous. $\times 100$.

the original surviving fibres have lost all the enzyme activity, whereas the regenerating myotubes show intense SDH activity (Fig. 11). By 25-30 days, these regenerating muscle fibres have recovered an almost mature looking histochemical profile for SDH activity (Figs. 12 and 13).

ATPase. ATPase activity also follows a similar developmental course; that is, it is reduced or lost completely in degenerating fibres by $8-10$ days while the regenerating fibres stain intensely for this enzyme (Fig. 14). However, as are the fibres in the

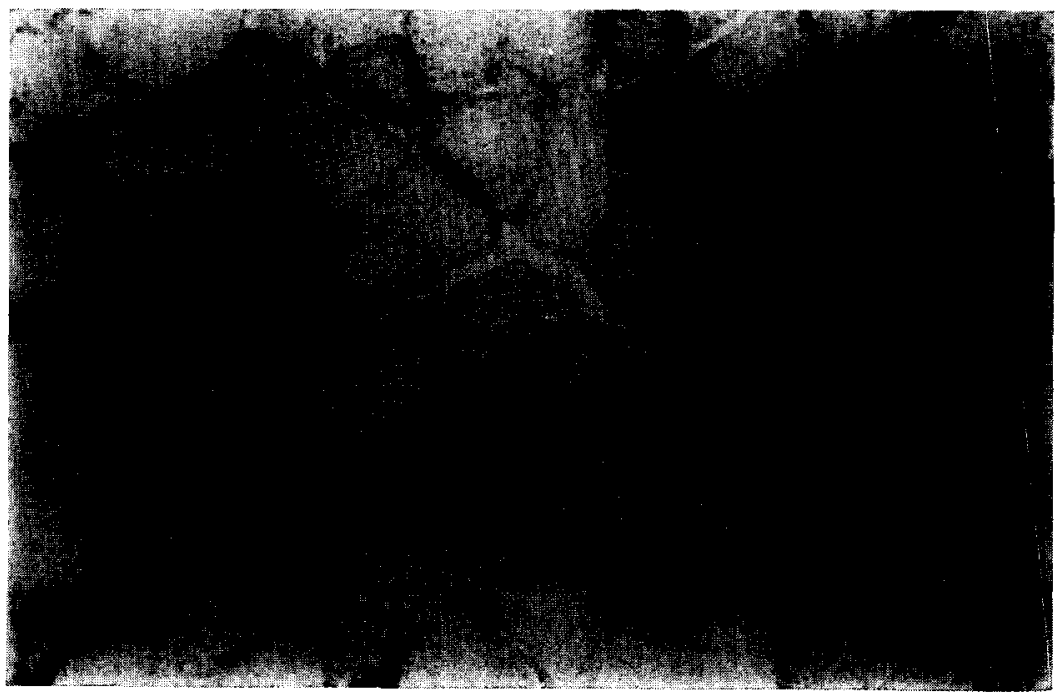

Fig. 13. SDH activity pattern in a control gastrocnemius muscle. $\times 100$. 


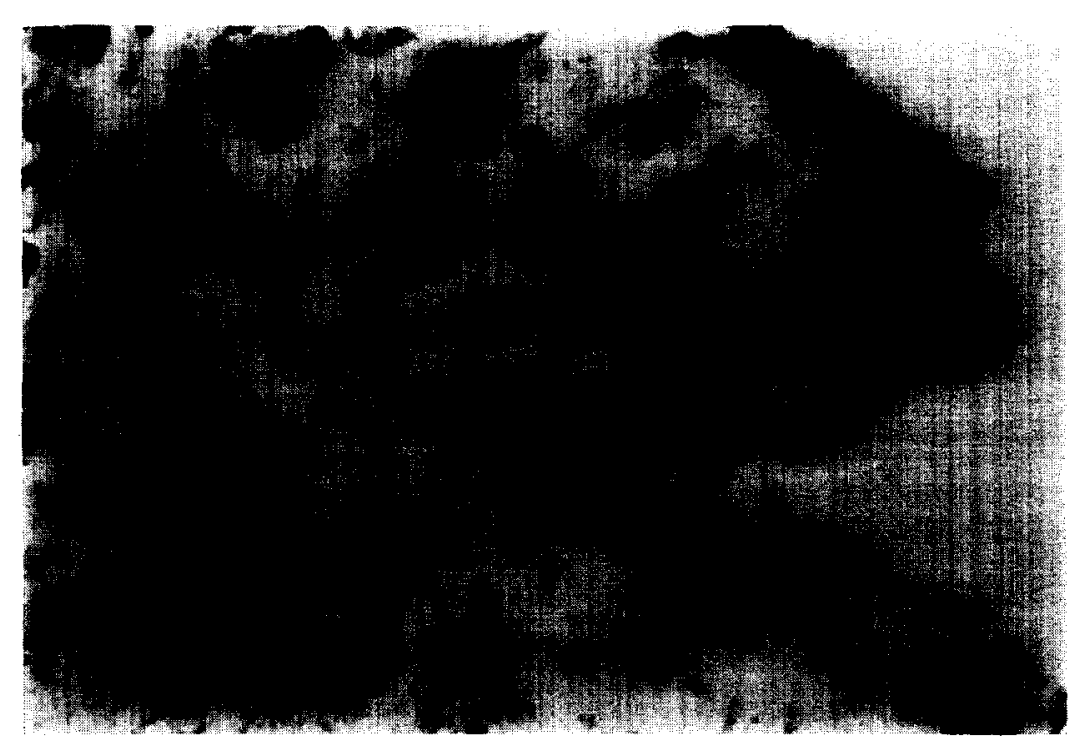

Fig. 14. Strong ATPase reaction in almost all of the regenerating myotubes in a 10-day control implant. $\times 100$.

control muscles, all regenerating fibres remain positively stained until the 50-day stage studied (Fig. 15 and 16).

Cholinesterase. The cholinesterase activity in relation to motor end-plates in the implanted, minced muscle fibres persists for 6-8 days, but it is completely lost by 12 days. In the meantime it appears in relation to the regenerating myotubes. It is diffused at the beginning, but by 25-30 days the motor end-plates become quite well defined with almost normal looking cholinesterase activity (Figs. 17 and 18).

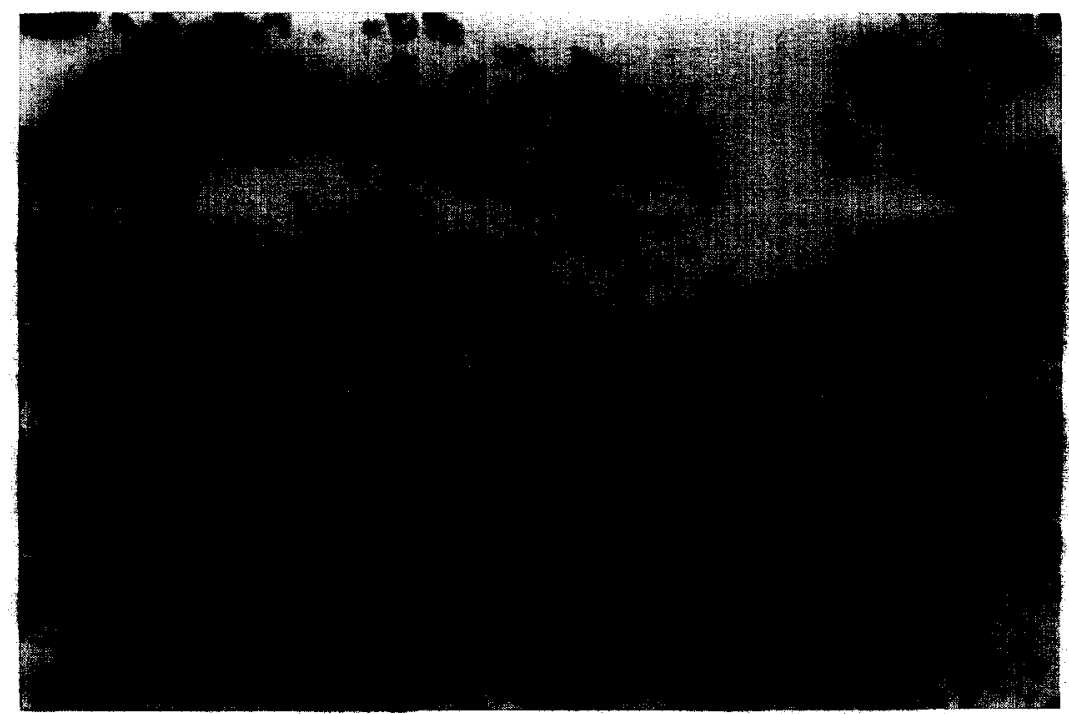

Fig. 15. All ATPase-positive muscle fibres in a 50-day control implant, $\times 100$. 


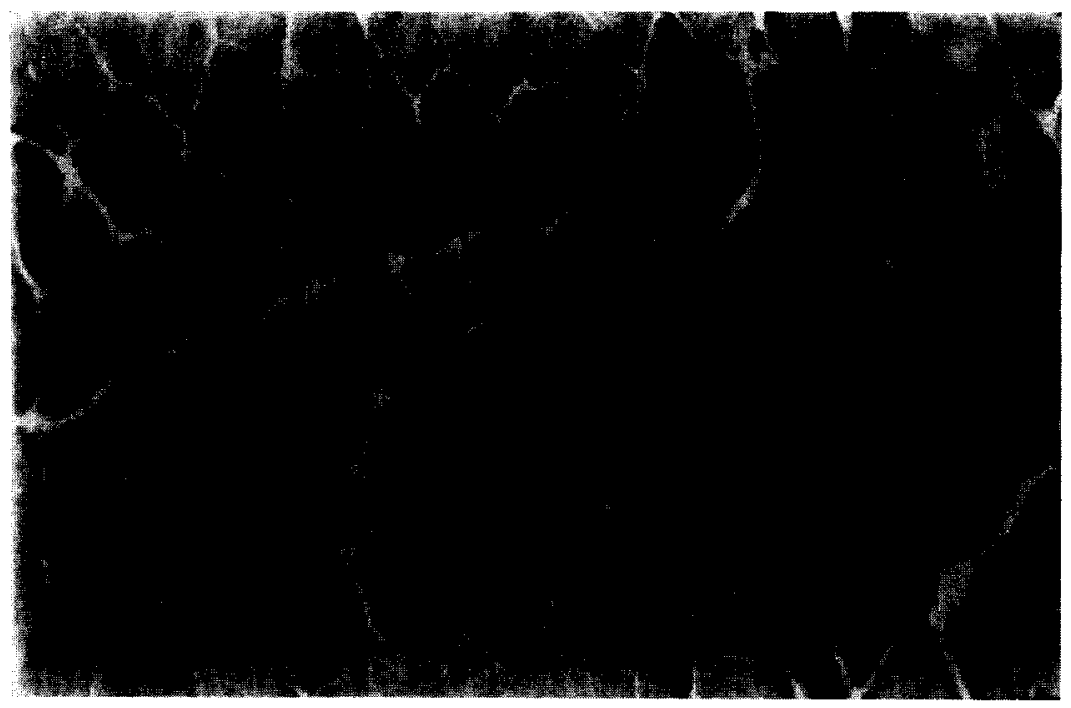

Fig. 16. ATPase activity in a control gastrocnemius muscle. Note that all fibres are ATPase-positive. $\times 100$.

\section{Denervated series}

$S D H$. By 6-8 days, the implanted degenerating fibres gradually lose their activity whereas by $6-10$ days the regenerating myotubes show rather intense SDH activity (Fig. 19). By 13-15 days, there is a gradual loss of enzymatic activity as well as a haphazard distribution of this enzyme. By 16-18 days there are left only a few regenerating fibres which show a faint and abnormal staining reaction for SDH (Fig. 20).

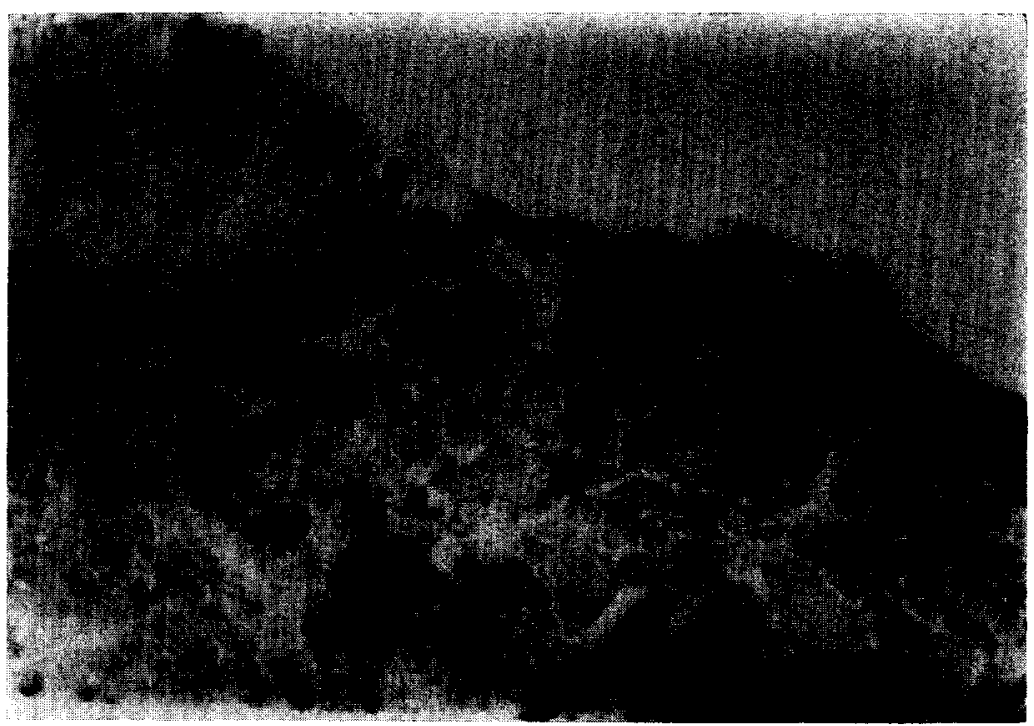

Fig. 17. Well defined cholinesterase activity (arrow) in relation to regenerating muscle fibres in a $35-$ day control implant, $\times 100$. 


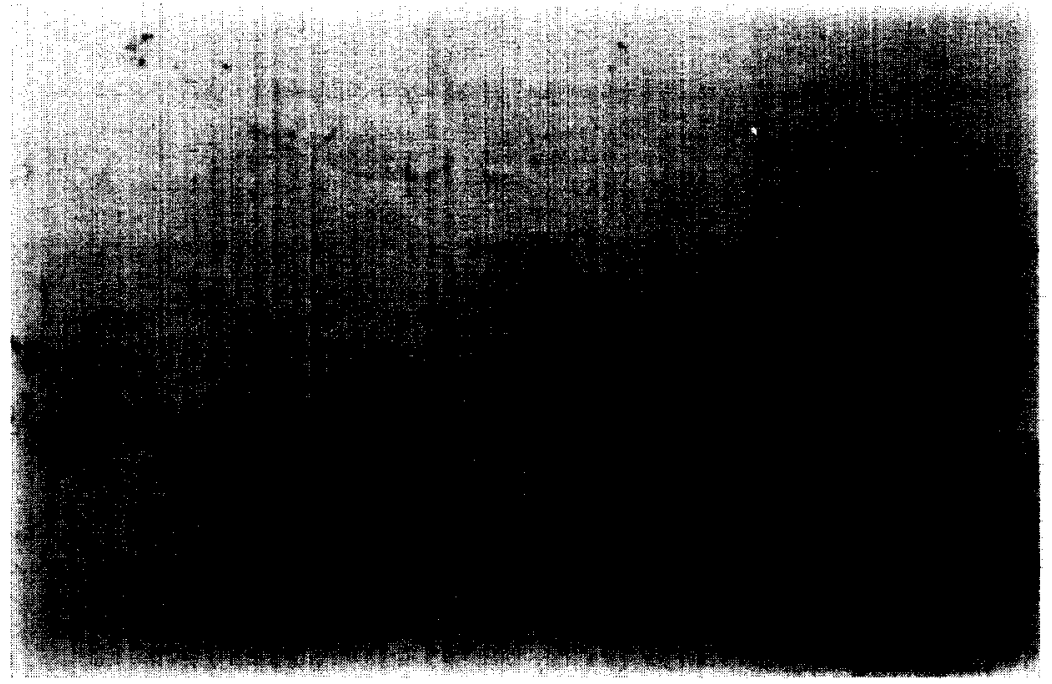

Fig. 18. Cholinesterase activity in a control gastrocnemius muscle. $\times 100$.

ATPase. ATPase activity also shows a pattern similar to SDH. The original fibres lose this enzyme by 6-10 days. The regenerating fibres show it initially (Fig. 21), but gradually degenerate with a corresponding loss of enzymatic activity.

Cholinesterase. The degenerating original fibres lose cholinesterase activity by 6-8 days, but the regenerating fibres do not develop any cholinesterase activity until 18-20 days, by which time there are few fibres remaining.

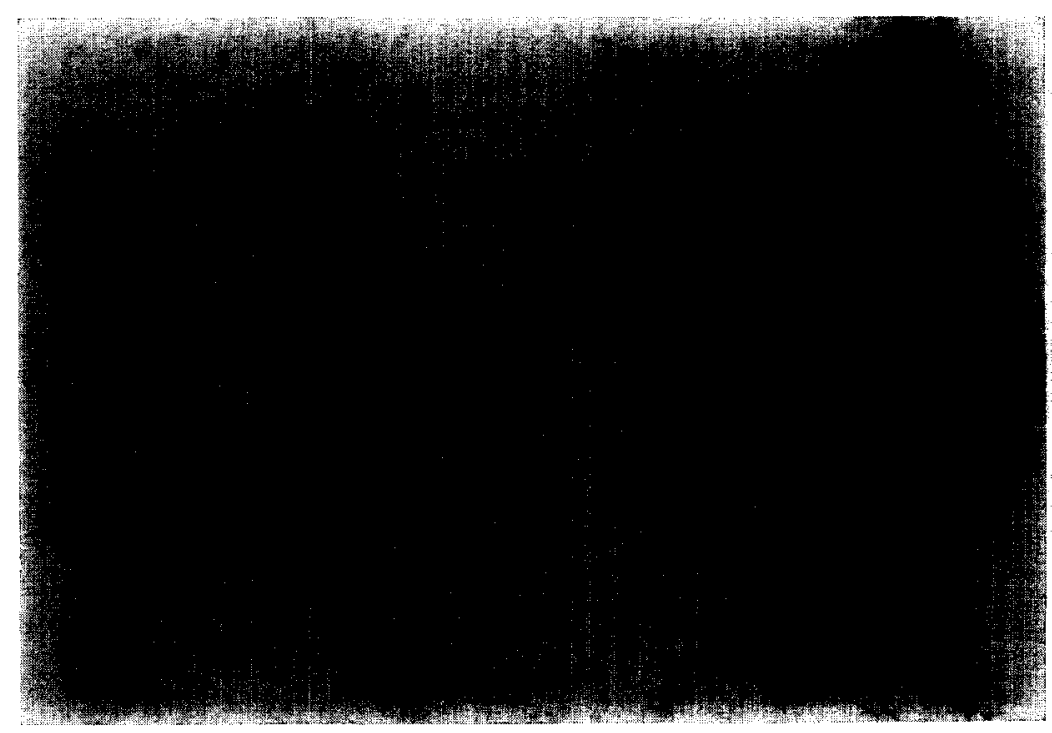

Fig. 19. Regenerating myotubes showing rather intense SDH activity in a 6-day denervated implant. $\times 100$. 


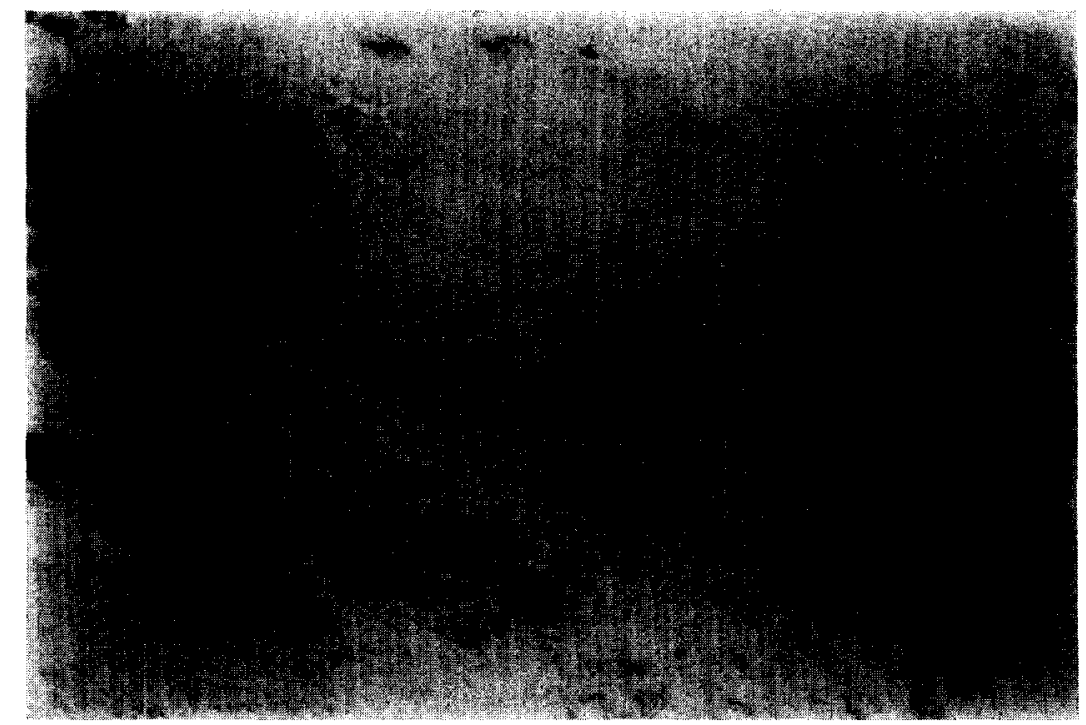

Fig. 20. Degenerating muscle fibres in a 16-day denervated implant. Some of these still show SDH activity. $\times 100$.

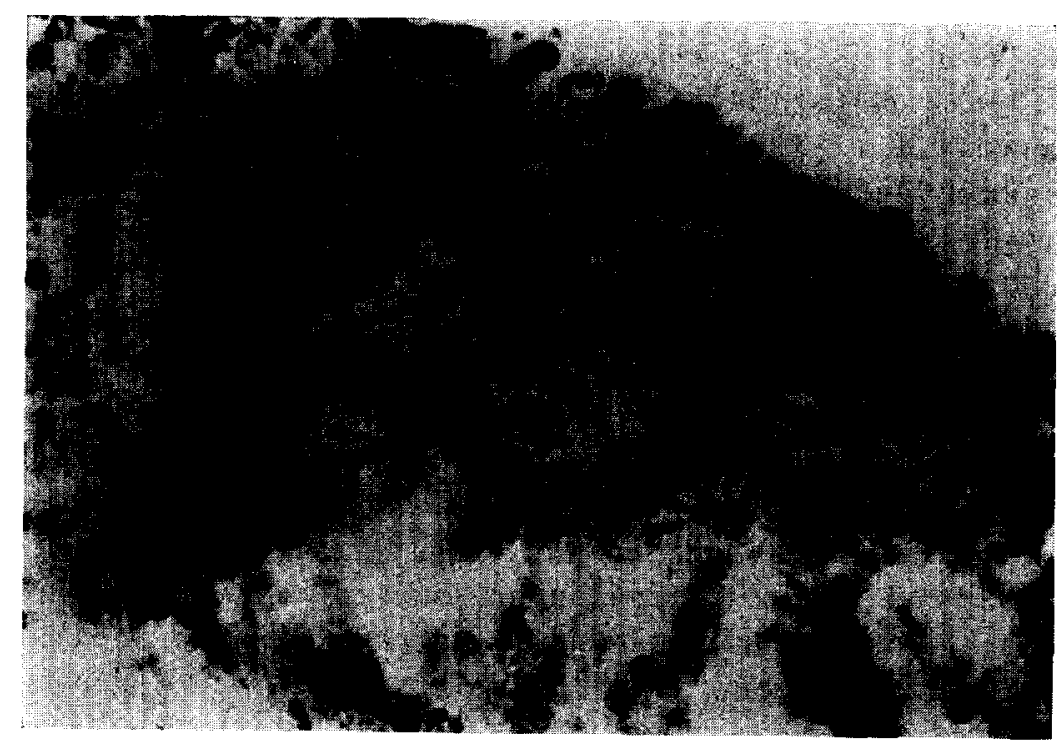

Fig. 21. Most of the regenerating muscle fibres showing ATPase activity in a 10-day denervated implant. $\times 100$.

\section{DISCUSSION}

The present investigation supports earlier observations (Hsu 1974; Mong 1975) that muscle regeneration can proceed to a considerable extent without innervation. However, the degree of muscle differentiation achieved before the onset of degeneration varies in various cases and is of considerable interest. For example Mong (1975), 
while working on triceps surae of rats, described a rather similar pattern of muscle regeneration in normal and denervated rats until day 30 . In both cases well-differentiated muscle fibres with distinct striations and peripheral nuclei were observed before regressive changes started in the denervated implant. In frogs, Hsu (1974) saw only early regeneration taking place in the denervated implants. Regenerated muscle fibres developed only faint striations and had mostly central nuclei when the degeneration of these fibres set in by the end of the 2 nd week. In the present series of experiments, the onset of degeneration of regenerating muscle fibres was much more rapid. It started by day 4 and was quite conspicuous by day 6 . The regenerating fibres never developed distinct cross-striations, and they had chains of central nuclei when degeneration set in. Why such closely related animals as rats and mice differ so much in terms of degree of muscle regeneration in the denervated condition is difficult to explain. It could be due to species differences, the metabolic pattern of the muscle involved or the life span of various animals (Hnik and Skorpie 1962). In this regard it is even more interesting to note that some workers have found quite different results working with the same animals. For example, Studitsky (1963) described very little or no muscle regeneration in the denervated condition in rats while Zhenevskaya (1962) described muscle regeneration up to the myotube stage in the denervated condition, followed by degeneration. Yeasting (1969) saw no difference whatsoever between denervated or normally innervated minced gastrocnemius muscle implants in mice during the 5 weeks of study. It is difficult to interpret such variation in results. These differences could be a result of local differences in the strains of rats and mice used or, more probably, due to the operational procedures adopted. Partial or incomplete denervation and/or secondary infection of the regenerate may cause these variations in results. From the description of denervation reported by Yeasting (1969), it seems he did not achieve a complete denervation.

The morphological picture of muscle degeneration following initial regeneration was similar to that described by Hsu (1974) and Mong (1975). The sarcoplasm became vacuolated, the striations disappeared and the nuclei became pyknotic, disc-shaped and packed together. The degeneration proceeded quickly, and practically all fibres had undergone complete degeneration by 3 weeks. At this time there remained only a bundle of connective and adipose tissue and in some cases only a thread-like band of connective tissue. This is quite different from what was observed in rats, where although there was a gradual increase in connective and adipose tissues, some atrophying muscle fibres remained viable for a long time (until about day 150) (Mong 1975).

Due to practically complete denervation, it is obvious that the animal could not use its denervated leg, i.e. it remained permanently flexed. It has been seen in many cases that disuse may result in atrophy of the muscle (Riley and Allin 1973: Tomanek and Lund 1974). However, Hsu (1971) observed that immobilization (by femurectomy) did not affect the minced muscle regeneration in frogs. More control experiments have to be performed to resolve the effect of disuse in cases of denervated minced muscle regeneration.

Histochemically, the present observations were similar to the ones made by Mong (1975). In his studies on rats, both normal and denervated implants showed 
SDH and myofibrillar ATPase activities during the early phases of regeneration. This was also the case in mice, except that in denervated implants, due to an early degeneration of the regenerating fibres, the histochemical differentiation of the muscle fibres did not proceed any further. However, most of the degenerating muscle fibres tended to retain positive histochemical reactions until the end.

Positive SDH and myofibrillar ATPase reactions were also observed in denervated muscle fibres in many earlier studies, both in vitro and in vivo (Engel and Karpati 1968; Askanas, Shafiq and Milhorat 1972). There was, however, observed a lack of histochemical differentiation of fibre types in these studies as well as in the denervated regenerating muscle fibres in rats (Mong 1975; Carlson and Gutmann 1976). Because of an early degeneration of the regenerated muscle fibres, caution must be exercised in using the mouse as a model for studies on myogenic properties of denervated regenerating or transplanted muscle.

From the present study, therefore, it seems clear that nerves are not required during the early stages of muscle regeneration in mice, but they are needed for further differentiation and maturation of these fibres and in the realization of normal physiological activities. It seems that the degree of muscle differentiation achieved in the denervated condition and the time of onset of muscle degeneration is variable in various species of animals and should be considered during further experimental studies.

\section{REFERENCES}

Askanas, V., S. A. Shafiq and A. T. Milhorat (1972) Histochemistry of cultured aneural chick muscle - Morphological maturation without differentiation of fiber types, Exp. Neurol., 37: 218-230.

Buller, A. J., J. C. Eccles and R. M. Eccles (1960) Interactions between motorneurons and muscles in respect of the characteristic speed of their responses, J. Physiol. (Lond.), 150: 417-439.

Burke, R. E., D. N. Levine, F. E. Fajac, P. Tsairis and W. K. Engel (1971) Mammalian motor units - Physiological and histochemical correlations in three types in a gastrocnemius, Science, 174: $709-711$.

Carlson, B. M. (1968) Regeneration of completely excised gastrocnemius muscle in the frog and rat from minced muscle fragments, J. Morphol., 125: 447-471.

Carlson, B. M. (1973) The regeneration of skeletal muscle - A review, Amer. J. Anat., 137: 119-150.

Carlson, B. M. and E. Gutmann (1976) Contractile and histochemical properties of sliced muscle grafts regenerating in normal and denervated rat limbs, Exp. Neurol., 50: 319-329.

Close, R. (1964) Dynamic properties of fast and slow skeletal muscles of the rat during development, J. Physiol. (Lond.), 173: 74-95.

Engel, W. K. and G. Karpati (1968) Impaired skeletal muscle maturation following neonatal neurectomy, Develop. Biol., 17: 713-723.

Guth, L., F. J. Samaha and R. W. Albers (1970) The neural regulation of some phenotypic differences between the fiber types of mammalian skeletal muscle, J. neurol. Sci., 15: 27-33.

Gutmann, E. and J. Zelena (1962) Morphological changes in the denervated muscle. In: E. Gutmann (Ed.), The Denervated Muscle, Publishing House of Czech. Acad. Sci., Prague, pp. 57-102.

Hamburger, V. (1939) The development and innervation of transplanted limb primordia of chick embryos, J. exp. Zool., 80: 347-390.

Harrison, R. G. (1904) An experimental study of the relation of the nervous system to the developing musculature in the embryo of the frog, Amer. J. Anat., 3: 197-202.

Hnik, P. and J. Skorpie (1962) Fibrillation activity in denervated muscle. In: E. Gutmann (Ed.), The Denervated Muscle, Publishing House of the Czech. Acad. Sci., Prague, pp. 136-150.

Hsu, L. (1971) The Role of Nerves in the Regeneration of Minced Muscle in Adult Anurans, Ph. D. thesis, University of Michigan, Ann Arbor. 
Hsu, L. (1974) The role of nerves in the regeneration of minced skeletal muscle in the adult Anurans Anat. Rec., 179: 119-136.

Hughes, A. F. W. (1968) Aspects of Neural Ontogeny, Lagos Press, London.

Mong, F. S. (1975) Histological and Histochemical Studies on the Nervous Influence on Minced Muscle Regeneration of Triceps surae of the Rat, Ph. D. thesis, University of Michigan, Ann Arbor.

Nachlas, M. M., K. C. Tsou, E. deSouza, C. S. Cheng and A. M. Seligman (1957) Cytochemical demonstration of succinic dehydrogenase by the use of a new p-nitraphenyl subsituted ditetrazole, J. Histochem. Cytochem., 5: 420-436.

Padykula, H. A. and E. Herman (1955) The specificity of the histochemical method for adenosine triphosphatase, J. Histochem. Cytochem., 3: 170-195.

Pearse, A. G. E. (1972) Histochemistry, Vol. 2, Churchill Livingstone, London.

Riley, D. A. and E. F. Allin (1973) The effect of inactivity, programmed stimulation, and denervation on the histochemistry of skeletal muscle fiber types, Exp. Neurol., 40: 391-413.

Samaha, F. J., L. Guth and R. W. Albers (1970) The neural regulation of gene expression in the muscle cell, Exp. Neurol., 27: 276-282.

Studitsky, A. N. (1963) Dynamics of development of myogenic tissue under conditions of explantation and transplantation. In: G. G. Rose (Ed.), Cinemicrography in Cell Biology, Academic Press, New York, N.Y., pp. 171-200.

Tomanek, R. J. and R. D. Lund (1974) Degeneration of different types of skeletal muscle fibers, Part 2 (Immobilization), J. Anat. (Lond.), 118:531-541.

Yeasting, R. A. (1969) The Effect of the Nerve Supply on the Regeneration of Minced Skeletal Muscle in the Mouse, $\mathrm{Ph}$. D. thesis, University of Louisville, $\mathrm{Ky}$.

Zhenevskaya, R. P. (1962) Experimental histologic investigation of skeletal muscle tissue, Rev. Canad. Biol., 21 : 457-470. 